\title{
Is Iterative Reconstruction an Improvement Over Filtered Back Projection in Processing Gated Myocardial Perfusion SPECT?
}

ARTICLE in THE OPEN MEDICAL IMAGING JOURNAL · OCTOBER 2008

DOI: $10.2174 / 1874347100802010017$

CITATIONS

2

7 AUTHORS, INCLUDING:

Kyoung Sook Won

Keimyung University

41 PUBLICATIONS 197 CITATIONS

SEE PROFILE

Salman Gohar

Carilion Clinic

6 PUBLICATIONS 40 CITATIONS

SEE PROFILE
READS

41

Martha Vallejo Mar

University of Texas MD Anderson Cancer Center 10 PUBLICATIONS 162 CITATIONS

SEE PROFILE

William Erwin

University of Texas MD Anderson Cancer Center

82 PUBLICATIONS 1,600 CITATIONS

SEE PROFILE 


\title{
Is Iterative Reconstruction an Improvement Over Filtered Back Projection in Processing Gated Myocardial Perfusion SPECT?
}

\author{
Kyoung Sook Won ${ }^{1,2}$, E. Edmund $\mathrm{Kim}^{1}$, Martha Mar ${ }^{1}$, Salman Gohar ${ }^{1}$, William Erwin ${ }^{1}$, Wei Wei ${ }^{3}$ and \\ Isis Gayed ${ }^{*}, 1$ \\ ${ }^{I}$ Department of Nuclear Medicine, The University of Texas M.D. Anderson Cancer Center, Houston, Texas, USA \\ ${ }^{2}$ Department of Nuclear Medicine, Keimyung University Dongsan Medical Center, Daegu, South Korea \\ ${ }^{3}$ Division of Quantitative Science, The University of Texas M.D. Anderson Cancer Center, Houston, Texas, USA
}

\begin{abstract}
The impact of iterative reconstruction (IR) on myocardial perfusion imaging (MPI) interpretation and functional results is under investigated. We evaluated the effect of IR on the interpretation and functional results of MPI compared with filtered back-projection (FBP). Material and Methods: Sixty patients with previously acquired MPI were randomly selected. Studies were processed using FBP and 2D ordered-subsets expectation-maximization (OSEM). Two physicians interpreted the images after IR and FBP processing. Results were confirmed by coronary angiography and/or clinical course. Left ventricular ejection fraction (LVEF), end-diastolic volume (EDV) and end-systolic volume (ESV) were calculated with IR and FBP. Results: The sensitivity, specificity, positive and negative predictive values and accuracy of physician 1 were $95 \%, 87 \%, 80 \%, 97 \%$, and $90 \%$ with FBP and $100 \%, 80 \%, 72 \%, 100 \%$, and $87 \%$ with IR. For physician 2, they were $95 \%, 95 \%, 91 \%, 97 \%$, and $95 \%$ with FBP and $81 \%, 95 \%, 90 \%, 90 \%$, and $90 \%$ with IR. There were no statistically significant differences between physicians' interpretations ( $P=0.71$ for FBP and 0.09 for IR). There was good correlation between IR and FBP calculated LVEF $(\mathrm{r}=0.98)$, EDV ( $\mathrm{r}=0.99)$, and ESV $(\mathrm{r}=0.99)$ but significant difference per patient $(P=0.02,0.03$ and 0.02 , respectively). Conclusion: There is no statistically significant difference in interpretation of MPI with IR versus FBP but there were statistically significant differences in functional results.
\end{abstract}

Keywords: Myocardial perfusion scintigraphy, SPECT, Iterative reconstruction, Filtered back-projection, SPECT reconstruction algorithms.

\section{INTRODUCTION}

All common SPECT camera software packages allow image reconstruction of gated myocardial perfusion studies using filtered back-projection (FBP). The most important benefit of FBP is rapid processing with contrast enhancement compared to planar scintigrams. However, some artifacts can be caused by this FBP algorithm, such as count spillover from adjacent extracardiac activity into the myocardium due to the blurring effects of the imaging system resolution and the reconstruction filter. Researchers have described a variety of iterative reconstruction (IR) methods as alternatives to the FBP algorithm. These methods include the simultaneous IR technique, the algebraic reconstruction technique, the maximum-likelihood expectation maximization algorithm, and the ordered--subsets expectation maximization (OSEM) algorithm [1-6]. These were developed and proposed as alternatives to FBP in heart, bone, and brain SPECTs [7-11]. These techniques aim to minimize artifacts related to system resolution, attenuation and scatter. They also aim to improve contrast and image resolution, and allow management of noise propagation through the use of regularization techniques. In the past, the process of IR required a large number of iterations in order to increase the reconstruction accuracy, explaining the excessive computational power demand of this technique and limiting their routine use. With

\footnotetext{
*Address correspondence to this author at the Department of Nuclear Medicine, The University of Texas M.D. Anderson Cancer Center, Houston, Texas, USA; E-mail: gayed@di.mdacc.tmc.edu
}

the increasing processing power, speed and memory of computer systems and the accelerated processing algorithms, the use of IR has become more widespread. IR techniques were previously reported to provide more accurate reconstruction than FBP in the case of sparse or incomplete data sets [12]. However, there is still uncertainty about the impact of replacement of the reconstruction algorithm on clinical imaging conditions, for example, those in gated myocardial perfusion SPECT.

There are few clinical reports in the literature on the efficacy of clinical application of IR techniques in gated myocardial perfusion SPECT in small number of patients $[13,14]$. Additionally, none of the studies evaluated the effect of IR on functional results compared to FBP. The purpose of our study was to evaluate the impact of IR using 2D OSEM on gated myocardial perfusion SPECT studies in comparison with FBP with regards to image interpretation, and functional results in sufficient number of patients and over a wide range of left ventricular ejection fractions (LVEFs).

\section{MATERIALS AND METHODS}

Upon approval of institutional review board, sixty patients who previously underwent gated myocardial perfusion SPECT scans using ${ }^{99 \mathrm{~m}} \mathrm{Tc}$--tetrofosmin with a wide range of LVEFs were randomly selected and included in the study. Among these patients, 20 had a history of coronary artery disease (CAD), 10 underwent myocardial perfusion imaging (MPI) as preoperative evaluation for cancer resection, 7 had myocardial infarctions, 5 had non-ischemic cardiomy- 
opathies, 4 post-stent insertions evaluation, 3 had coronary artery bypass grafts, and 2 had congestive heart failure.

\section{Imaging Procedure}

The patients were instructed to discontinue taking any calcium channel blockers or beta-blockers and avoid consuming caffeine for $24 \mathrm{~h}$ prior to undergoing a stress test. All patients underwent a single-day rest and stress protocol. On the day of the test, each patient was injected with $370 \mathrm{MBq}$ of ${ }^{99 \mathrm{~m}} \mathrm{Tc}$--tetrofosmin at rest. SPECT images were acquired 30 min later using a dual head gamma camera (e. cam Signature Series; Siemens Medical Solutions, Hoffman Estates, IL). Three hours later, 47 patients underwent pharmacologic stress testing using either adenosine (43) or dobutamine (4), and 13 exercised on a treadmill, according to standard stress protocols. ${ }^{99 \mathrm{~m}} \mathrm{Tc}$ tetrofosmin $1110 \mathrm{MBq}$ was injected at peak stress; this was followed by a 20 to 30 min rest period, during which the patient was allowed to drink juice and eat crackers. Stress gated SPECT images were subsequently obtained. The rest and stress SPECT acquisition parameters were: $180^{\circ}$ arc, 64 views, $25 \mathrm{~s} /$ view, $64 \times 64$ matrix, and 1.45 zoom, with 8 intervals for gated stress acquisition. The raw data were processed using FBP reconstruction with a Butterworth filter; order of 5 and a cut-off frequency of $0.5 \mathrm{Ny}-$ quist $(0.76 \mathrm{cycles} / \mathrm{cm})$. The raw data were processed again using a 2D OSEM algorithm incorporating compensation for system resolution, 2 subsets and 12 iterations, and an $8.4 \mathrm{~mm}$ full-width at half maximum 3D post-reconstruction Gaussian filter.

\section{Image Interpretation}

Two experienced nuclear medicine physicians who were blinded to the clinical data evaluated the SPECT images with regard to perfusion findings. Each physician evaluated the images twice; one time after the images were processed using FBP and a second time after the images were processed using IR. The images were interpreted at different times and in no particular order. Both physicians were blinded to their own previous interpretations of images processed by the other reconstruction method. Perfusion findings were recorded by each physician on a 13 left ventricular segments model. The perfusion abnormalities were scaled on a sixpoint semiquantitative scale: $0=$ normal, $1=$ mild ischemia, $2=$ moderate ischemic, $3=$ severe ischemia, $4=$ scar, and 5 $=$ mixed scar and ischemia. In six patients with discordant readings, normal interpretation using both processing methods by one physician versus abnormal interpretation using both processing methods by the other, were resolved in a final consensus interpretation by the two physicians together. The accuracy of the interpretation was established after correlation of the perfusion findings with those of coronary angiography performed within 4 weeks interval, past history of $\mathrm{CAD}$ and/or recurrence of ischemic event during follow-up for at least 6 months. Abnormal perfusion (1 to 5 on the semiquantitative scale) was considered as true positive for $\mathrm{CAD}$ if there was significant $(>50 \%)$ coronary obstruction on coronary angiography or if there was perfusion defect more than one segment with regional wall motion abnormality on gated SPECT with or without history of MI. Normal perfusion was considered as true negative if coronary angiography was normal or there was no past history of CAD and no ischemic events during six-month follow-up. Functional Quantitative Analysis

Functional results of left ventricle obtained using quantitative gated SPECT (QGS, Cedars-Sinai Medical Center, Los Angeles, California, USA), which consisted of LVEF, end-diastolic volume (EDV), and end-systolic volume (ESV), were obtained after the FBP and IR processing methods were applied. The results were collected and compared between IR and FBP.

\section{Statistical Analysis}

All continuous variables are expressed as mean $\pm \mathrm{SD}$. Interpretation of perfusion findings were compared between physicians for each modality using a Fisher's exact test. Comparisons between modalities within each physician and inference concerning disagreement rates were also made using the same test. The credible intervals for differences in proportion were listed for each segment and overall CAD status. LVEFs, EDVs, ESVs for both processing methods were compared and differences analyzed statistically using descriptive, correlation, and non-parametric Wilcoxon signed-rank test to compare these variables in pairs. Additionally, Bland-Altman plots of the differences in functional measurements with each reconstruction method from the mean were also performed. All tests were two-sided with pvalues of 0.05 or less considered statistically significant. All analyses were carried out in SAS version 9 (SAS institution, Cary, NC). All plots were produced using S-Plus 7.0 (Insightful, Seattle, WA).

\section{RESULTS}

There were 28 male patients and 32 female patients in the study group. Their mean age was $64 \pm 10 \mathrm{y}$.

\section{MPI Interpretations}

Physician 1 interpreted 25 studies as abnormal and 35 as normal using FBP and 29 studies as abnormal and 31 as normal using IR. In comparison, physician 2 interpreted 22 studies as abnormal and 38 as normal using FBP and 19 as abnormal and 41 studies as normal using IR. There were no statistically significant differences between the interpretations of the two physicians according to the method of image reconstruction ( $P=0.71$ for FBP and $P=0.09$ for IR). In 10 patients, coronary angiography confirmed the accuracy of the interpretation. Nine of 10 coronary angiographies showed CAD and the remaining one showed normal coronary arteries.

Twelve of the remaining 50 patients had history of CAD and fixed perfusion defects with corresponding regional wall motion abnormalities on the gated myocardial perfusion SPECT. Thus, a total of 21 patients were regarded as having CAD.

The distribution of true positive, false positive, true negative or false negative perfusion studies according to the reconstruction methods is shown for both physicians in (Table 1). IR yielded higher interpretation disagreements between the physicians in 10 patients (17\%) than FBP did, which yielded the disagreements in only 3 patients $(5 \%)$, though this difference was not statistically significant. As determined by physician 1, the sensitivity, specificity, positive predictive value (PPV), negative predictive value (NPV) and 
accuracy of MPI were $95 \%, 87 \%, 80 \%, 97 \%$, and $90 \%$, respectively, for FBP and $100 \%, 80 \%, 72 \%, 100 \%$, and $87 \%$, respectively, for IR. As determined by physician 2, the sensitivity, specificity, PPV, NPV and accuracy of MPI were $95 \%, 95 \%, 91 \%, 97 \%$, and $95 \%$, respectively, for FBP and $81 \%, 95 \%, 90 \%, 90 \%$, and $90 \%$, respectively, for IR.

Table 1. Comparison of MPI Interpretation Using FBP Versus IR by the Two Physicians

\begin{tabular}{|c|c|c|c|c|c|c|c|c|}
\hline \multirow{3}{*}{} & \multicolumn{6}{|c|}{ Number of Patients (\%) } \\
\cline { 2 - 9 } & \multicolumn{6}{|c|}{ FBP } & \multicolumn{5}{c|}{ IR } \\
\cline { 2 - 9 } & TP & FP & TN & FN & TP & FP & TN & FN \\
\hline \hline Physician 1 & $20(33)$ & $5(8)$ & $34(57)$ & $1(2)$ & $21(35)$ & $8(13)$ & $31(52)$ & $0(0)$ \\
\hline Physician 2 & $20(33)$ & $2(3)$ & $37(62)$ & $1(2)$ & $17(28)$ & $2(3)$ & $37(62)$ & $4(7)$ \\
\hline$P$ value & 1 & 0.4 & 0.71 & 1 & 0.56 & 0.09 & 0.36 & 0.1 \\
\hline
\end{tabular}

We then evaluated the disagreements between the two physicians for each myocardial segment (Table 2). Among total 780 segments, the number of disagreement was 85 $(11 \%)$ for FBP and $103(13 \%)$ for IR $(P=0.19)$. The anterior, inferior, and apical inferior segments had the highest number of disagreements between the two physicians using IR, whereas the anterior, apical-anterior and apical-inferior segments had the highest number of disagreements using FBP (Fig. 1A,B). After adjusting for the number of comparisons examined, none of these differences approached statistical significance.

\section{Functional Results}

The mean LVEF, EDV, and ESV were $49.7 \% \pm 16.5 \%$, $123.5 \pm 71.3 \mathrm{ml}$, and $71.1 \pm 60.1 \mathrm{ml}$, respectively, using FBP and $50.4 \% \pm 17.7 \%, 119.9 \pm 66.5 \mathrm{ml}$, and $71.0 \pm 61.4 \mathrm{ml}$, respectively, using IR.

There was good correlation in the quantitative functional results between the two reconstruction methods, with $\mathrm{r}=$ 0.98 for LVEF and r= 0.99 for each of EDV and ESV (Fig. 2). However, there was a significant difference between FBP and IR in the individual measurements per patient, with $P=0.02$ for LVEF, $P=0.03$ for EDV, and $P=0.02$ for ESV. BlandAltman analysis demonstrates wider differences between the results of each reconstruction method from the mean obtained with both methods (Fig. 3). The Bland-Altman plot mean \pm 2 standard deviation for LVEF, EDV, and ESV are- $0.9 \pm 4.6 \%$, $2.0 \pm 16.0 \mathrm{ml}$ and $2.1 \pm 13.2 \mathrm{ml}$, respectively. Higher LVEFs and higher EDVs tend to have wider spread around the mean of the two reconstruction methods.

\section{DISCUSSION}

Prior studies have demonstrated superior image quality with IR methods versus FBP $[7,10,15][16]$ however the impact of the improved image quality on image interpretation remains to be an intriguing question. This study compared the impact of IR versus FBP on image interpretation and functional results of gated MPI in 60 patients with wide range for LVEFs. Our results indicated that there was no statistically significant difference in image interpretation and findings using IR versus FBP. This is in agreement with previous study evaluating the effects of IR on brain imaging which demonstrated ability of improved separation of smaller structures in the brain but there was no significant difference in tracer binding and diagnostic information [10]. Another study by Zakavi et al. also showed no significant difference in the number of normal versus abnormal MPI studies reconstructed with FBP and OSEM after motion introduced artifacts [14]. Studies performed to compare myocardial F-18 flourodeoxyglucose uptake and myocardial ${ }^{13} \mathrm{~N}-$ ammonia perfusion with IR versus FBP in the PET arena,

Table 2. Number of Disagreements in Segmental Findings Using FBP and IR as Interpreted by Two Physicians

\begin{tabular}{|c|c|c|c|c|}
\hline $\begin{array}{c}\text { Myocardial } \\
\text { Segment }\end{array}$ & $\begin{array}{c}\text { Number of Disagreements } \\
\text { Using FBP }\end{array}$ & $\begin{array}{c}\text { FBP Disagreements Rate \% } \\
\text { (Exact 95\% CI*) }\end{array}$ & $\begin{array}{c}\text { Number of Disagreements } \\
\text { Using IR }\end{array}$ & $\begin{array}{c}\text { IR Disagreements Rate \% } \\
\text { (Exact 95\% CI) }\end{array}$ \\
\hline \hline Anterior & 9 & $15(7-27)$ & 13 & $22(12-33)$ \\
\hline Anteroseptal & 7 & $12(5-23)$ & 6 & $10(4-19)$ \\
\hline Septal & 7 & $12(5-23)$ & 4 & $13(6-23)$ \\
\hline Inferoseptal & 3 & $5(1-14)$ & 12 & $7(2-14)$ \\
\hline Inferior & 5 & $8(3-18)$ & 6 & $20(11-31)$ \\
\hline Inferolateral & 5 & $12(5-23)$ & 9 & $10(4-19)$ \\
\hline Lateral & 8 & $13(6-25)$ & 9 & $15(7-25)$ \\
\hline Anterolateral & 3 & $5(1-14)$ & 7 & $15(7-25)$ \\
\hline Apical & 7 & $12(5-23)$ & 7 & $12(5-21)$ \\
\hline Apical-anterior & 9 & $15(7-27)$ & 4 & $12(5-21)$ \\
\hline Apical-septal & 5 & $8(3-18)$ & 12 & $7(2-14)$ \\
\hline Apical-inferior & 10 & $17(8-29)$ & 8 & $20(11-31)$ \\
\hline Apical-lateral & 8 & $13(6-25)$ & & $13(6-23)$ \\
\hline
\end{tabular}


(A)

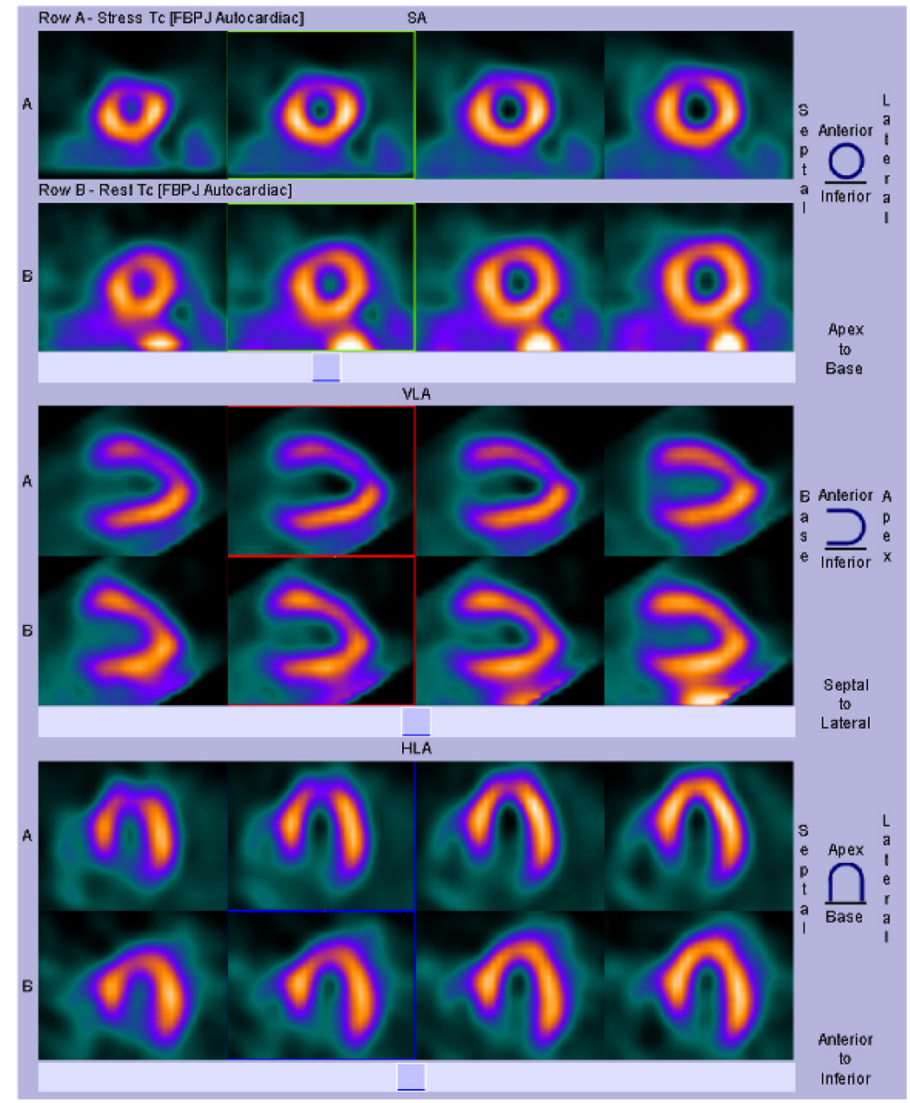

(B)

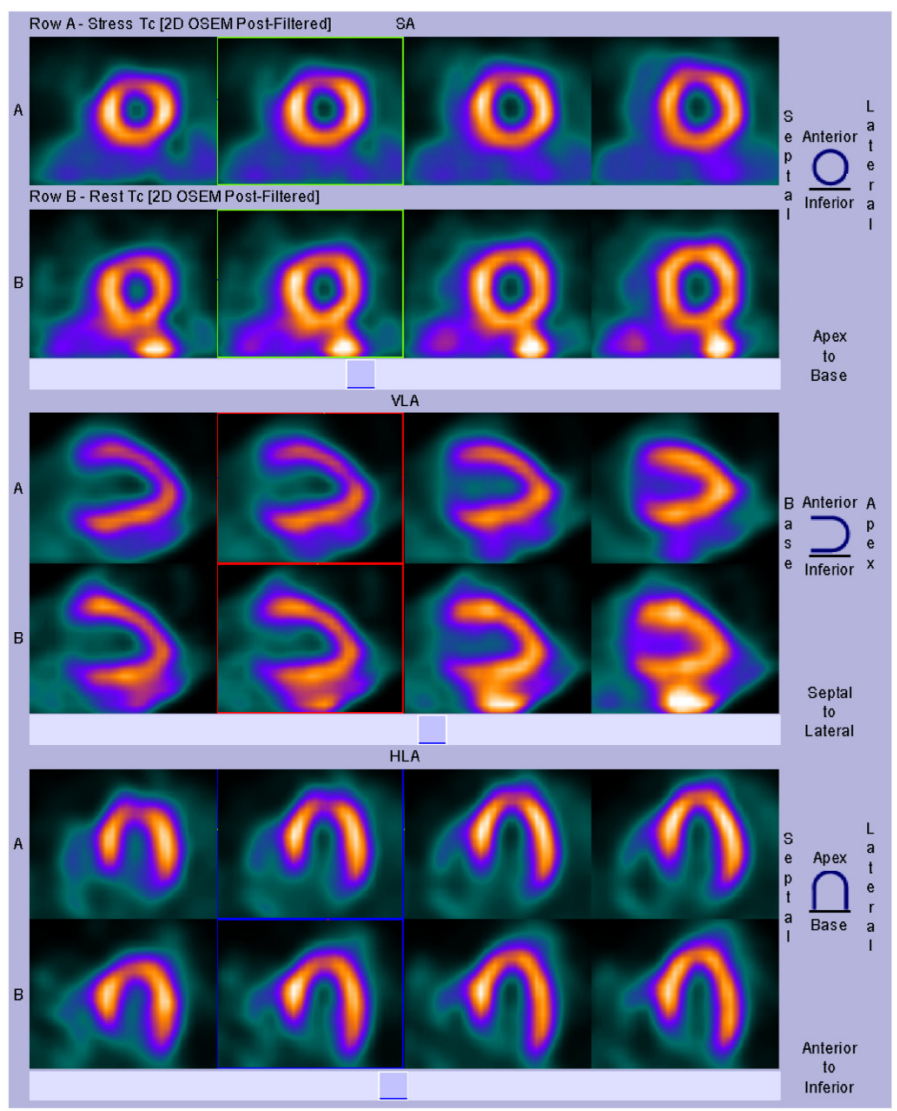

Fig. (1). (A) Moderate ischemia is seen in the apical anterior and anterior myocardial segments on FBP images. (B) myocardial perfusion is near normal on IR images. Coronary angiography two days later proved normal coronaries. 
(A)

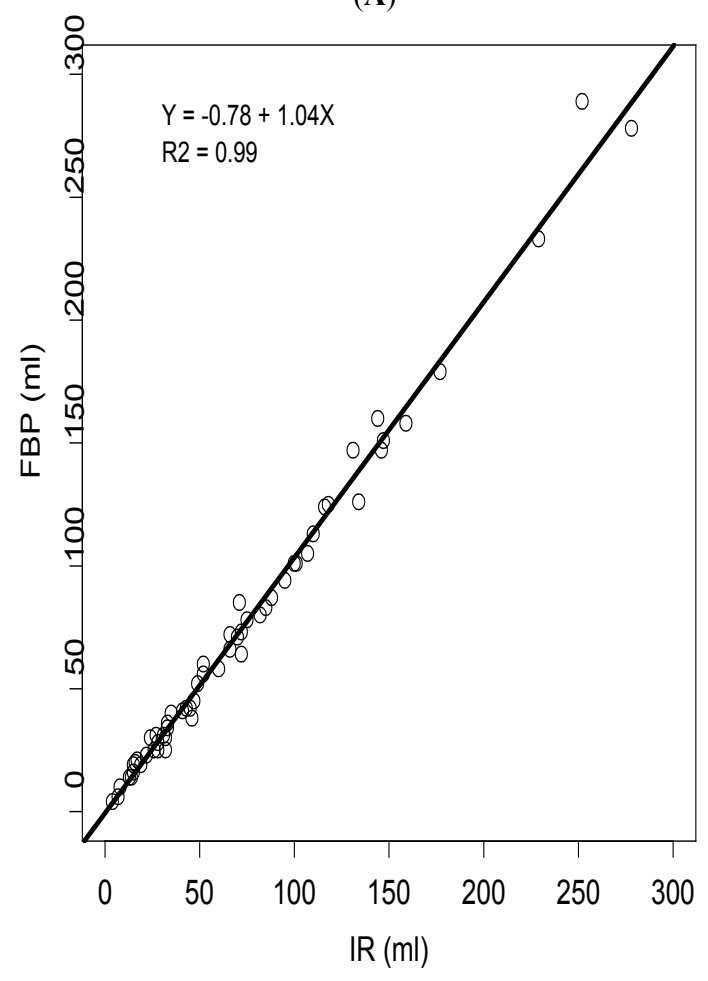

(B)

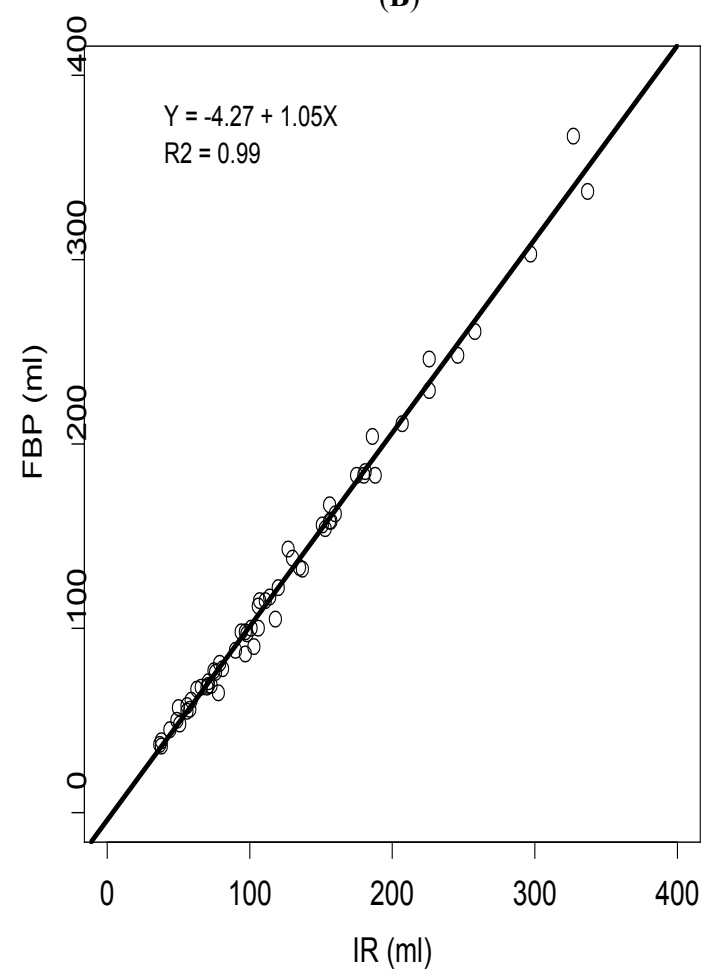

(C)

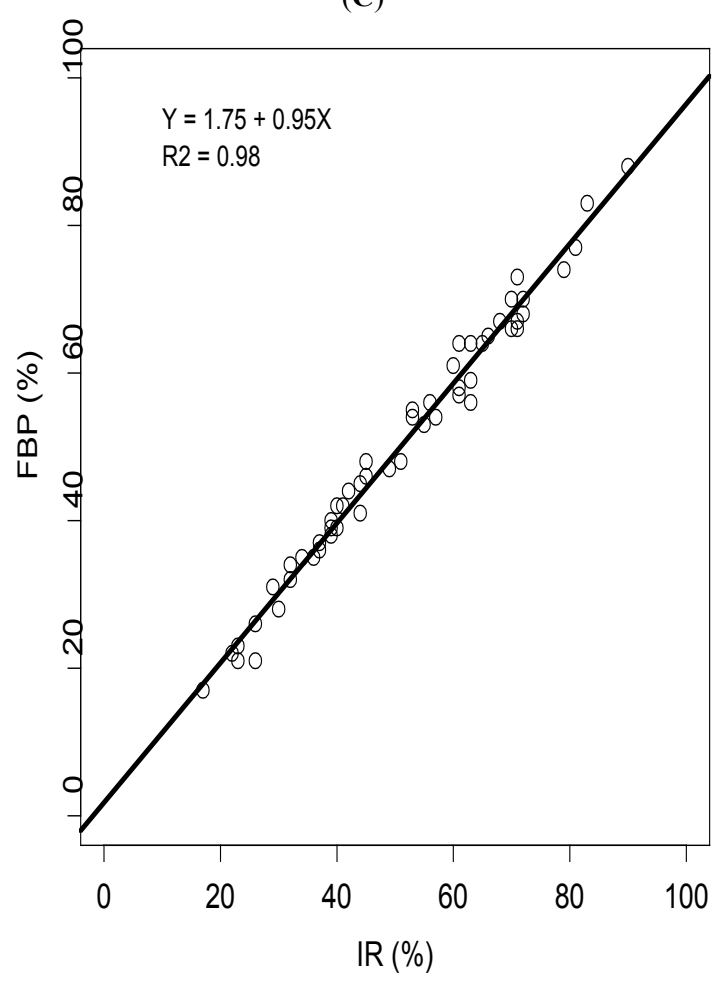

Fig. (2). Regression plots of ESVs (A), EDVs (B) and LVEF (C) calculated using both IR and FBP show excellent correlation.

also demonstrated no significant difference between the two reconstruction methods $[15,17]$.

IR readings had higher disagreements (17\%) between the two physicians than FBP (5\%). This may be explained by the fact that both physicians are more experienced reading gated
MPI using FBP than IR. This may suggest that an initial learning curve is expected if IR is adopted to process gated MPI. Our results are different from a previous study conducted by Pretorius et al. where they reported a significant improvement in accuracy of detecting coronary artery disease using combined compensation of attenuation, scatter 


\section{Bland Altman Plots on the Raw Scale}
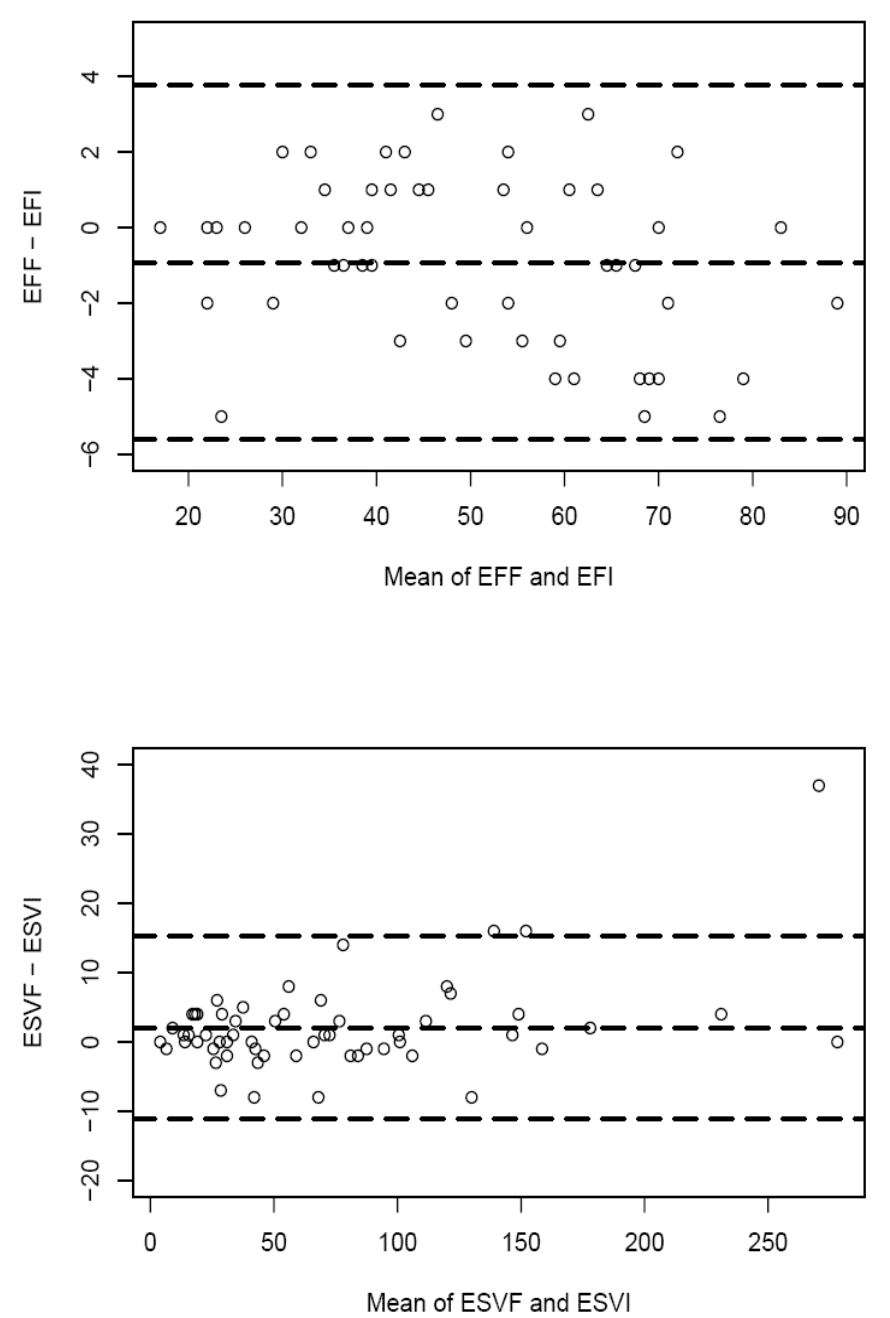

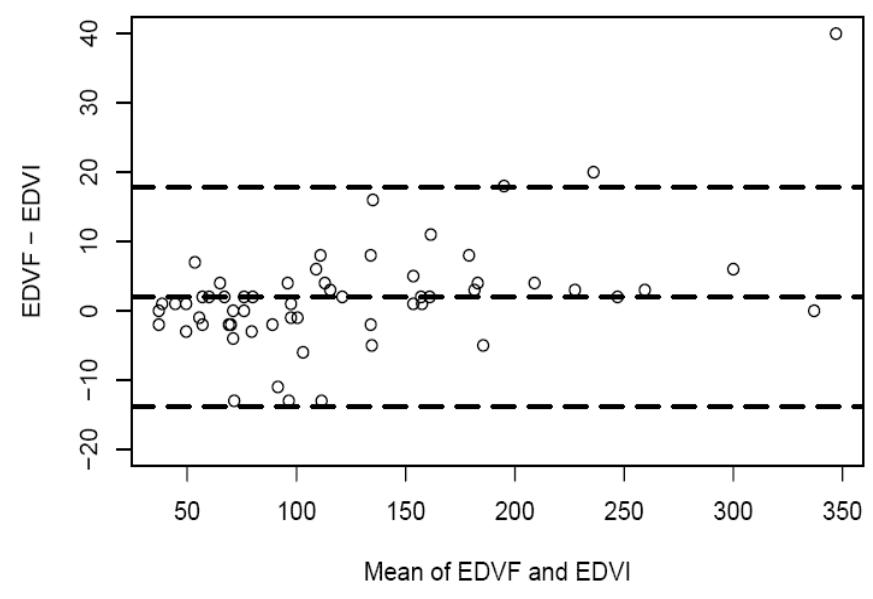

EFF : ejection fraction filtered back projection

EFI : ejection fraction iterative reconstruction

EDVF : end diastolic volume filtered back projection

EDVI : end diastolic volume iterative reconstruction

ESVF : end systolic volume filtered back projection

ESVI : end systolic volume iterative reconstruction

Fig. (3). Bland Altman Plots for the spread of LVEF, EDV and ESV measurements from the mean of IR and FBP result.

and resolution with IR over FBP $(P=0.018)$. The images were interpreted by 7 observers in Pretorius et al. study. Interestingly, when they analyzed the data for each observer separately, although IR was better, the difference was not statistically significant [13]. The difference between the study by Pretorius et al. and ours is that they compared multiple image improvement factors with IR (attenuation, scatter and resolution correction) against FBP while in our study we compared IR alone versus FBP. Additionally, the improved accuracy for detection of CAD is cumulative for the 7 readers in their study. This may explain the better accuracy they noted in detection of CAD using IR over our results.

To our Knowledge, this is the first study to evaluate the effects of IR compared to FBP on functional results. There was good correlation of quantitative functional results including LVEF, EDV, and ESV between the two reconstruction modalities in our study. On the other hand, significant differences in the measurements per patient between the two methods were noted although the overall mean values were comparable. This suggest that functional results obtained by the two different processing methods can not be used for follow up or comparison of patient results at different times and they are not inter-changeable. Additionally, new normal values should be established with normal data base for functional measurements obtained with IR.

One limitation of our study is that no gold standard to evaluate which reconstruction method is producing more accurate functional results was available in this retrospective study. Future studies with large number of patients and with wide range of quantitative results are needed to compare the results of the two reconstruction methods against a gold standard e.g. magnetic resonance imaging or radionuclide ventriculography.

Since the processing time was slightly longer using IR versus FBP (1min versus $5 \mathrm{~min}$ ) with no significant impact on the accuracy of interpretation of MPI studies, we suggest that IR be reserved to special patients. This would probably include patients with significant underlying splanchnic activity or obese patients [10].

The highest segmental disagreement between the two reconstruction methods was mostly in the inferior, apicalinferior, anterior and apical-anterior segments. This is probably due to the effect of diaphragmatic and breast attenuation. This suggests that although image quality might be slightly better with IR it does not decrease the difficulties 
associated with attenuation artifacts. Therefore, for appreciable improvement in image quality and possibly image interpretation, we recommend the use of multiple correction algorithms, including attenuation, scatter and resolution correction in addition to IR rather than IR alone.

\section{CONCLUSION}

We found no statistically significant differences in the accuracy of myocardial perfusion interpretation between FBP and IR. On the other hand, there is significant difference between the functional results of gated MPI obtained with each processing method.

\section{REFERENCES}

[1] Gilbert P. Iterative methods for the three-dimensional reconstruction of an object from projections. J Theor Biol 1972; 36: 105-117.

[2] Gordon R, Bender R, Herman GT. Algebraic reconstruction techniques (ART) for three-dimensional electron microscopy and x-ray photography. J Theor Biol 1970; 29: 471-481.

[3] Shepp L, Vardi Y. Maximum likelihood reconstruction for emission tomography. IEEE Trans Med Imaging 1982; 1: 113-122.

[4] Nuyts J, Dupont P, Stroobants S, Maes A, Mortelmans L, Suetens P. Evaluation of maximum likelihood based attenuation correction in positron emission tomography. IEEE Trans Nucl Sci 1999; 46: 1136-1141.

[5] Lange K, Carson R. EM reconstruction algorithms for emission and transmission tomography. J Comput Assist Tomogr 1984; 8: 306316.

[6] Kauppinen T, Yang J, Kilpelainen H, Kuikka JT. Quantitation of neuroreceptors: a need for better SPECT imaging. Nuklearmedizin 2001; 40: 102-106.

[7] Kitchens CT, Halkar RK, Alazraki NP, Galt J. Difference in filtered-back projection and iterative reconstruction in a patient with increased splanchnic uptake. Clin Nucl Med 2005; 30: 623-624.

[8] Wells, RG, Farncombe T, Chang E, Nicholson RL. Reducing bladder artifacts in clinical pelvic SPECT images. J Nucl Med 2004; 45: 1309-1314.
[9] Starck SA, Ohlsson J, Carlsson S. An evaluation of reconstruction techniques and scatter correction in bone SPECT of the spine. Nucl Med Commun 2003; 24: 565-570.

[10] Koch W, Hamann C, Welsch J, Pöpperl G, Radau PE, Tatsch K. Is iterative reconstruction an alternative to filtered backprojection in routine processing of dopamine transporter SPECT studies? J Nucl Med. 2005; 46: 1804-1811.

[11] Kauppinen T, Koskinen MO, Alenius S, Vanninen E, Kuikka JT. Improvement of brain perfusion SPET using iterative reconstruction with scatter and non-uniform attenuation correction. Eur J Nucl Med 2000; 27: 1380-1386.

[12] Hatton RL, Hutton BF, Angelides S, Choong KKL, Larcos G. Improved tolerance to missing data in myocardial perfusion SPET using OSEM reconstruction. Eur J Nucl Med Mol Imaging 2004; 31: 857-861

[13] Pretorius PH, King MA, Gifford HC, et al. Receiver operating characteristic comparison of CAD detection accuracy of filtered backprojection reconstruction with all of the clinical imaging information available to physicians and solely stress slices iteratively reconstructed with combined compensation. J Nucl Cardiol 2005; 12: 284-293.

[14] Zakavi SR, Zonoozi A, Kakhki VD, Hajizadeh M, Momennezhad M, ARiana K. Image reconstruction using filtered backprojection and iterative method: effect on motion artifacts in myocardial perfusion SPECT. J Nucl Med Technol 2006; 34(4): 220-3.

[15] Schiepers C, Nuyts J, Hsiao-Ming W, Verma RC. PET with ${ }^{18} \mathrm{~F}$ flourine: effects of iterative versus filtered backprojection reconstruction on kinetic modeling. IEEE Trans Nucl Sci 1997; 44: 1593.

[16] Ghoarun S, Baete K, Nuyts J, Groenewald W, Dupont P. The influence of attenuation correction and reconstruction techniques on the detection of hypo-perfused lesions in brain SPECT images. Nucl Med Commun 2006; 27(10): 765-72.

[17] Sondergaard HM, Madson MM, Biosen K, et al. Evaluation of iterative reconstruction (OSEM) versus filtered back-projection for the assessment of myocardial glucose uptake and myocardial perfusion using dynamic PET. Eur J Nucl Med Mol Imaging 2007; 34(3): 320-9. 